Benchmark examples for model reduction of linear time invariant dynamical systems.

Chahlaoui, Younes and Van Dooren, Paul 2005

MIMS EPrint: 2008.9

Manchester Institute for Mathematical Sciences

School of Mathematics

The University of Manchester

\footnotetext{
Reports available from: http://eprints.maths.manchester.ac.uk/

And by contacting: The MIMS Secretary

School of Mathematics

The University of Manchester

Manchester, M13 9PL, UK
} 


\title{
Benchmark Examples for Model Reduction of Linear Time-Invariant Dynamical Systems
}

\author{
Younes Chahlaoui ${ }^{1}$ and Paul Van Dooren ${ }^{2}$ \\ 1 School of Computational Science, Florida State University, Tallahassee, U.S.A. \\ younes.chahlaoui@laposte.net \\ 2 CESAME, Université catholique de Louvain, Louvain-la-Neuve, Belgium \\ vdooren@csam.ucl.ac.be
}

Summary. We present a benchmark collection containing some useful real world examples, which can be used to test and compare numerical methods for model reduction. All systems can be downloaded from the web and we describe here the relevant characteristics of the benchmark examples.

\subsection{Introduction}

In this paper we describe a number of benchmark examples for model reduction of linear time-invariant systems of the type

$$
\left\{\begin{array}{l}
\dot{x}(t)=A x(t)+B u(t) \\
y(t)=C x(t)+D u(t)
\end{array}\right.
$$

with an associated transfer function matrix

$$
G(s)=C\left(s I_{N}-A\right)^{-1} B+D .
$$

The matrices of these models are all real and have the following dimensions : $A \in \mathbb{R}^{N \times N}, B \in \mathbb{R}^{N \times m}, C \in \mathbb{R}^{p \times N}$, and $D \in \mathbb{R}^{p \times m}$. The systems are all stable and minimal and the number of state variables $N$ is thus the order of the system. In model reduction one tries to find a reduced order model,

$$
\left\{\begin{array}{l}
\dot{\hat{x}}(t)=\hat{A} \hat{x}(t)+\hat{B} \hat{u}(t) \\
\hat{y}(t)=\hat{C} \hat{x}(t)+\hat{D} \hat{u}(t)
\end{array}\right.
$$

of order $n \ll N$, such that the transfer function matrix $\hat{G}(s)=\hat{C}\left(s I_{n}-\right.$ $\hat{A})^{-1} \hat{B}+\hat{D}$ approximates $G(s)$ in a particular sense, and model reduction methods differ typically in the error measure that is being minimized. In assessing the quality of the reduced order model, one often looks at the following characteristics of the system to be approximated 
- the eigenvalues of $A$ (or at least the closest ones to the $j \omega$ axis), which are also the poles of $G(s)$

- the controllability Gramian $\mathcal{G}_{c}$ and observability Gramian $\mathcal{G}_{o}$ of the system, which are the solutions of the Lyapunov equations

$$
A \mathcal{G}_{c}+\mathcal{G}_{c} A^{T}+B B^{T}=0, \quad A^{T} \mathcal{G}_{o}+\mathcal{G}_{o} A+C^{T} C=0
$$

- the singular values of the Hankel map - called the Hankel singular values $(\mathrm{HSV})$ - which are also the square-roots of the eigenvalues of $\mathcal{G}_{c} \mathcal{G}_{o}$

- the largest singular value of the transfer function as function of frequency - called the frequency response -

$$
\sigma(\omega)=\|G(j \omega)\|_{2} .
$$

These characteristics can be compared with those of the reduced order model $\hat{G}(s)$. Whenever they are available, we give all of the above properties for the benchmark examples we discuss in this paper. The data files for the examples can be recovered from $h t t p: / / w w w . w i n . t u e . n l / n i c o n e t / n i c o n e t . h t m l$. For each example we provide the matrix model $\{A, B, C, D\}$, and (when available) the poles, the Gramians, the Hankel singular values, a frequency vector and the corresponding frequency response. For more examples and additional details of the examples of this paper, we refer to [CV02]. Some basic parameters of the benchmarks discussed in the paper are given below.

\begin{tabular}{|c|l|c|c|c|c|}
\hline Section & Example (Acronym) & Sparsity & $N$ & $m$ & $p$ \\
\hline 2 & Earth Atmosphere (ATMOS) & no & 598 & 1 & 1 \\
\hline 3 & Orr-Sommerfeld (ORR-S) & no & 100 & 1 & 1 \\
\hline 4 & Compact Disc player (C-DISC) & yes & 120 & 2 & 2 \\
\hline 5 & Random (RAND) & yes & 200 & 1 & 1 \\
\hline 6 & Building (BUILD-I) & yes & 48 & 1 & 1 \\
\hline 6 & Building (BUILD-II) & yes & 52788 & 1 & 1 \\
\hline 6 & Clamped Beam (BEAM) & yes & 348 & 1 & 1 \\
\hline 7 & Intern. Space Station (ISS-I) & yes & 270 & 3 & 3 \\
\hline 7 & Intern. Space Station (ISS-II) & yes & 1412 & 3 & 3 \\
\hline
\end{tabular}

\subsection{Earth Atmospheric Example (ATMOS)}

This is a model of an atmospheric storm track [FI95]. In order to simulate the lack of coherence of the cyclone waves around the Earth's atmosphere, linear damping at the storm track's entry and exit region is introduced. The perturbation variable is the perturbation geopotential height. The perturbation equations for single harmonic perturbations in the meridional $(y)$ direction of the form $\phi(x, z, t) e^{i l y}$ are :

$$
\frac{\partial \phi}{\partial t}=\nabla^{-2}\left[-z \nabla^{2} D \phi-r(x) \nabla^{2} \phi\right]
$$




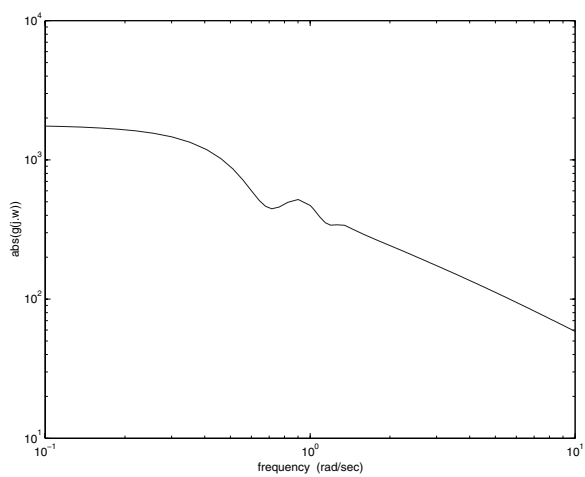

Fig. 24.1. Frequency response (ATMOS)
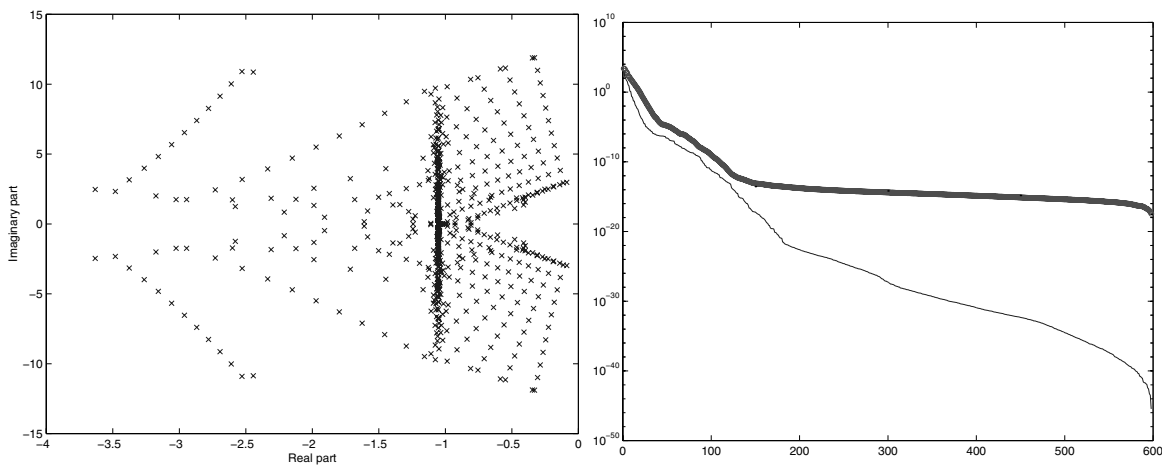

Fig. 24.2. Eigenvalues of $A$ (ATMOS) Fig. 24.3. $\cdots \operatorname{svd}\left(\mathcal{G}_{c}\right), \operatorname{orvd}\left(\mathcal{G}_{o}\right),-$ hsv

where $\nabla^{2}$ is the Laplacian $\frac{\partial^{2}}{\partial x^{2}}+\frac{\partial^{2}}{\partial z^{2}}-l^{2}$ and $D=\frac{\partial}{\partial x}$. The linear damping rate $r(x)$ is taken to be $r(x)=h\left(2-\tanh \left[\left(x-\frac{\pi}{4}\right) / \delta\right]+\tanh \left[\left(x-\frac{7 \pi}{2}\right) / \delta\right]\right)$. The boundary conditions are expressing the conservation of potential temperature (entropy) along the solid surfaces at the ground and tropopause:

$$
\begin{aligned}
& \frac{\partial^{2} \phi}{\partial t \partial z}=-z D \frac{\partial \phi}{\partial z}+D \phi-r(x) \frac{\partial \phi}{\partial z} \quad \text { at } \quad z=0, \\
& \frac{\partial^{2} \phi}{\partial t \partial z}=-z D \frac{\partial \phi}{\partial z}+D \phi-r(x) \frac{\partial \phi}{\partial z} \quad \text { at } \quad z=1 .
\end{aligned}
$$

The dynamical system is written in generalized velocity variables $\psi=$ $\left(-\nabla^{2}\right)^{\frac{1}{2}} \phi$ so that the dynamical system is governed by the dynamical operator:

$$
A=\left(-\nabla^{2}\right)^{\frac{1}{2}} \nabla^{-2}\left(-z D \nabla^{2}+r(x) \nabla^{2}\right)\left(-\nabla^{2}\right)^{-\frac{1}{2}}
$$

where the boundary equations have rendered the operators invertible. We refer to [FI95] for more details, including the type of discretization that was used. 


\subsection{Orr-Sommerfeld Equation (ORR-S)}

The Orr-Sommerfeld operator for the Couette flow in perturbation velocity variables is given by :

$$
A=\left(-D^{2}\right)^{\frac{1}{2}} D^{-2}\left(-i j k D^{2}+\frac{1}{R e} D^{4}\right)\left(-D^{2}\right)^{-\frac{1}{2}}
$$

where $D:=\frac{d}{d y}$ and appropriate boundary conditions have been introduced so that the inverse operator is defined. Here, Re is the Reynolds number and $k$ is the wave-number of the perturbation. This operator governs the evolution of $2-$ dimensional perturbations. The considered matrix is a $100 \times 100$ discretization for a Reynolds number $R e=800$ and for $k=1$. We refer to [FI01] for more details, including the type of discretization that was used.

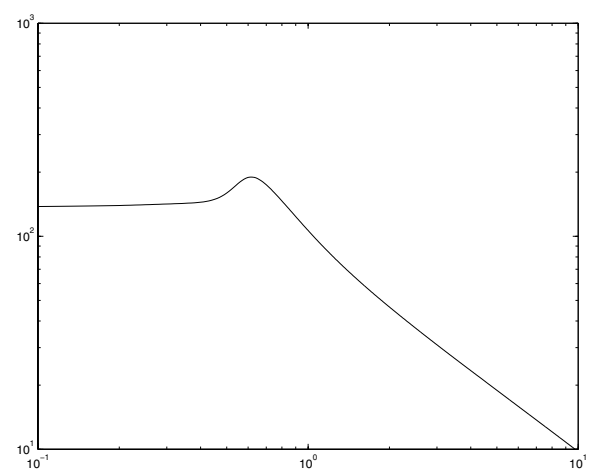

Fig. 24.4. Frequency response (ORR-S)

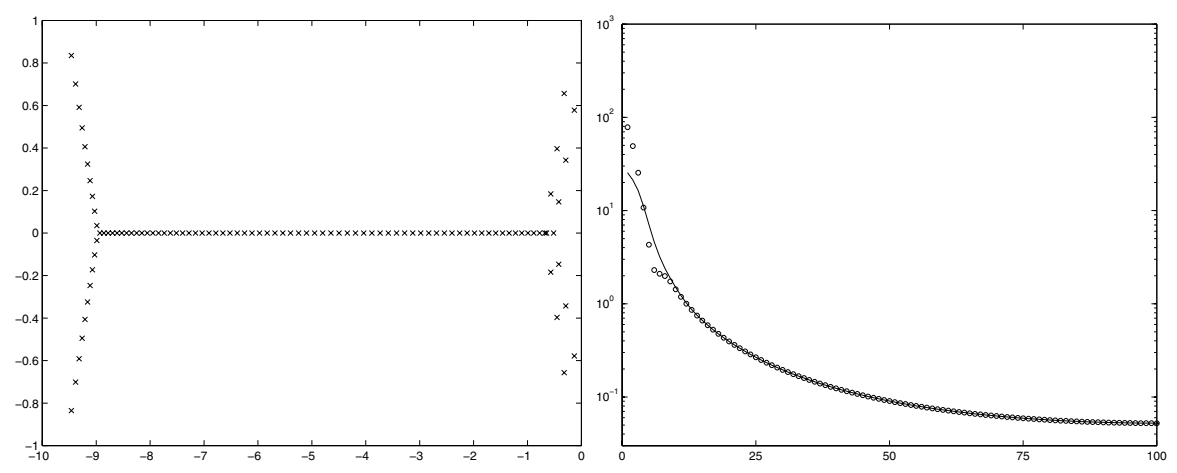

Fig. 24.5. Eigenvalues of $A(\mathrm{ORR}-\mathrm{S}) \quad$ Fig. 24.6. $\cdots \operatorname{svd}\left(\mathcal{G}_{c}\right), \operatorname{o~} \operatorname{svd}\left(\mathcal{G}_{o}\right),-\mathrm{hsv}$ 


\subsection{Compact Disc Player Example (C-DISC)}

The $C D$ player control task is to achieve track following, which amounts to pointing the laser spot to the track of pits on a CD that is rotating. The mechanism that is modeled consists of a swing arm on which a lens is mounted by means of two horizontal leaf springs. The rotation of the arm in the horizontal plane enables reading of the spiral-shaped disc-tracks, and the suspended lens is used to focus the spot on the disc. Since the disc is not perfectly flat and since there are irregularities in the spiral of pits on the disc, the challenge is to find a low-cost controller that can make the servo-system faster and less sensitive to external shocks. We refer to [DSB92, WSB96] for more details.

It is worth mentioning here that this system is already a reduced order model obtained via modal approximation from a larger rigid body model (which is a second order model).
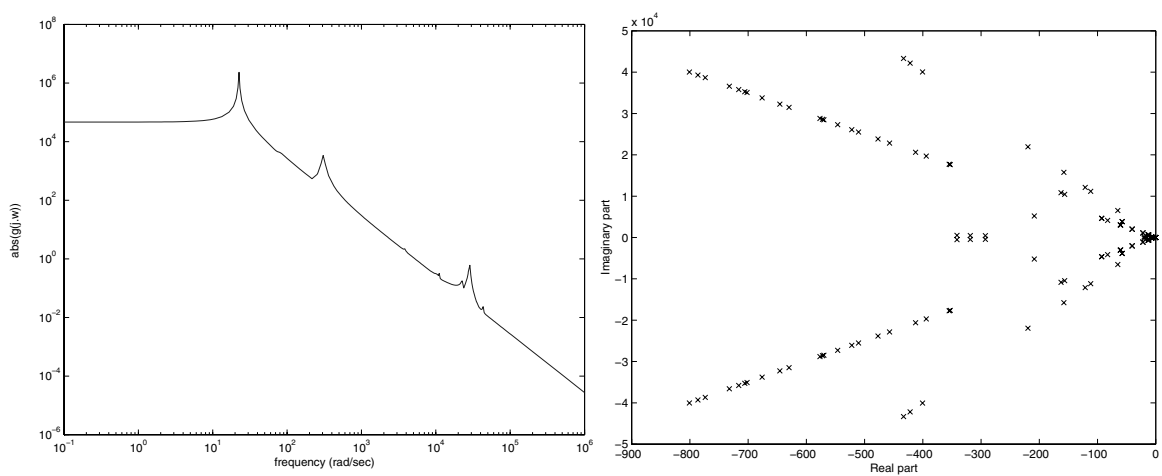

Fig. 24.7. Frequency response (C-DISC)

Fig. 24.8. Eigenvalues of $A$ (C-DISC)

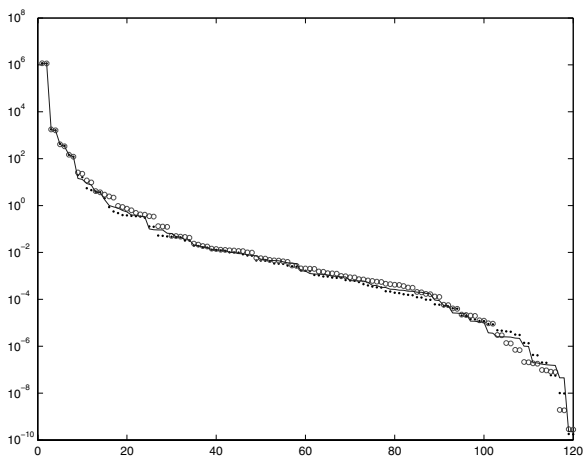

Fig. 24.9. $\cdots \operatorname{svd}\left(\mathcal{G}_{c}\right), o \operatorname{svd}\left(\mathcal{G}_{o}\right),-$ hsv

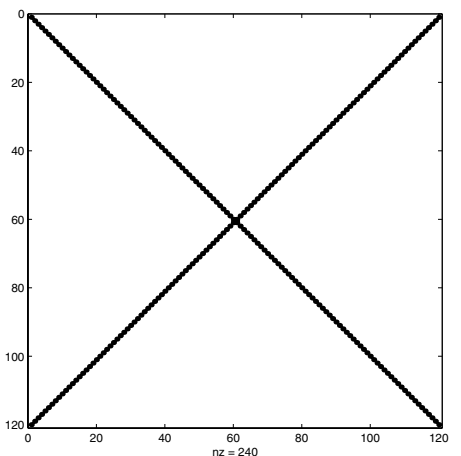

Fig. 24.10. Sparsity of $A$ (C-DISC) 

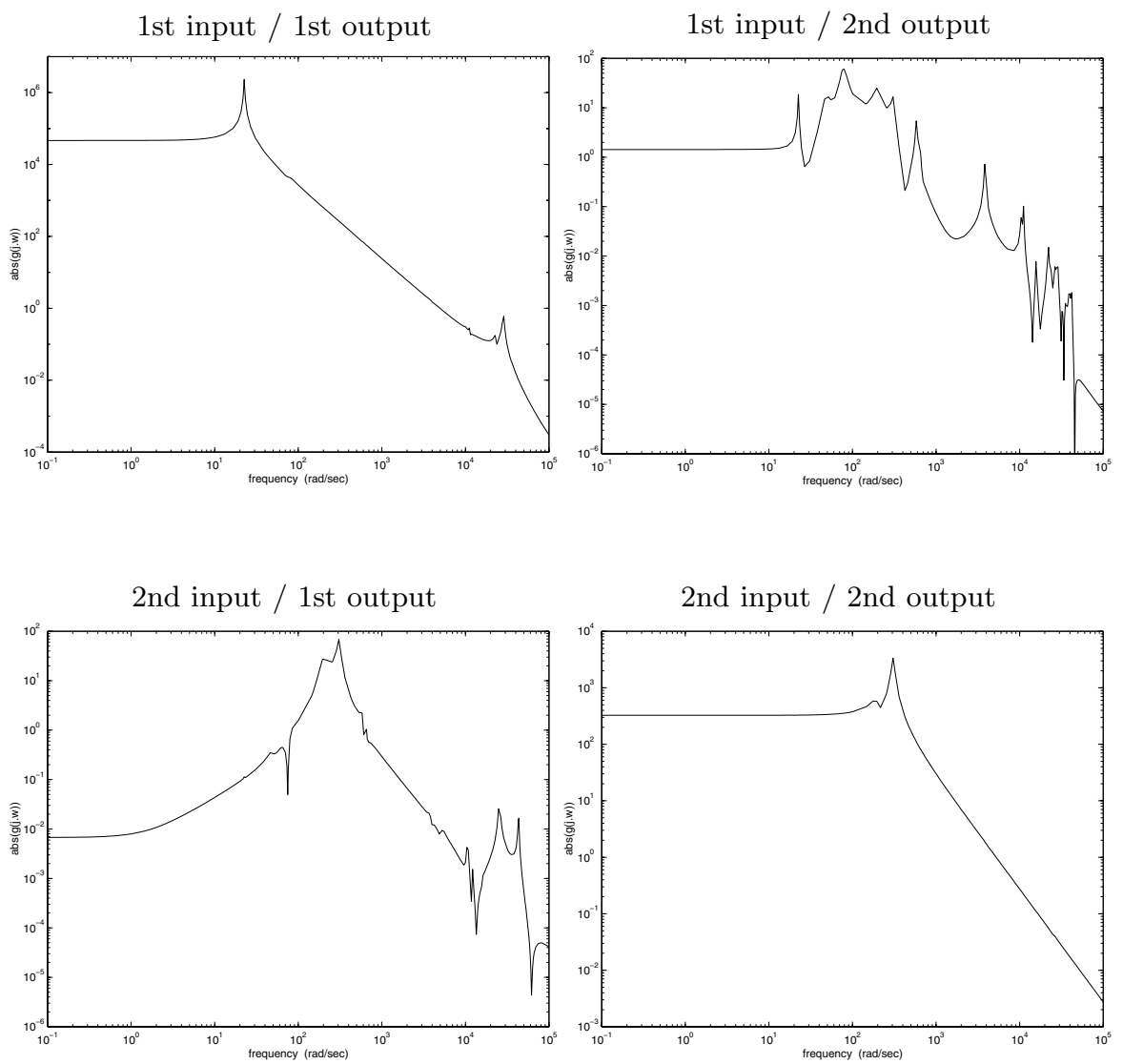

Fig. 24.11. Frequency responses of the 2-input 2-output system (C-DISC) 


\subsection{Random Example (RAND)}

This is a randomly generated example with an $A$ matrix that is sparse and stable, and has a prescribed percentage of nonzero elements. This is a simple example to approximate but it is useful to compare convergence rates of iterative algorithms. It is extracted from the Engineering thesis of V. Declippel [DeC97].
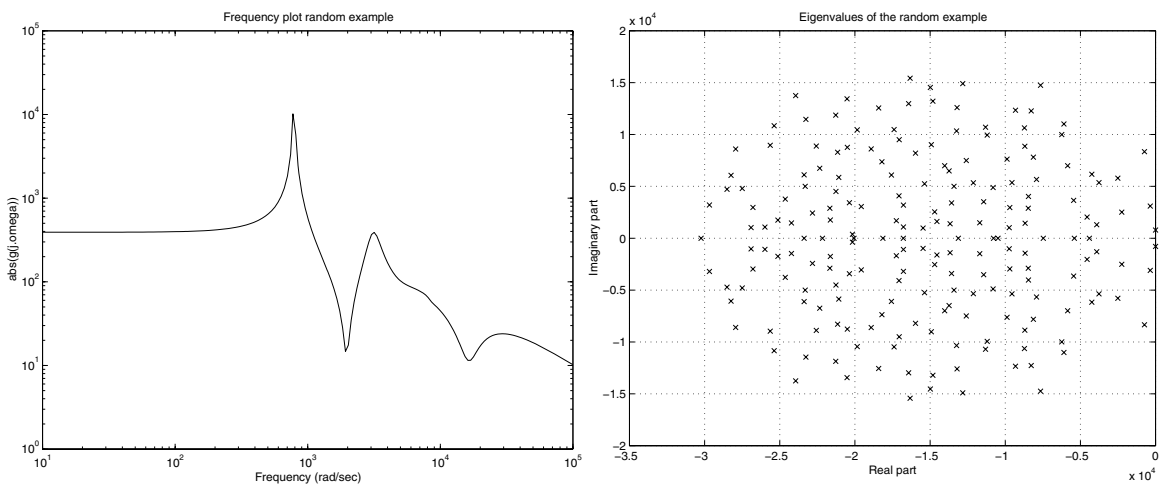

Fig. 24.12. Frequency response (RAND) Fig. 24.13. Eigenvalues of $A$ (RAND)

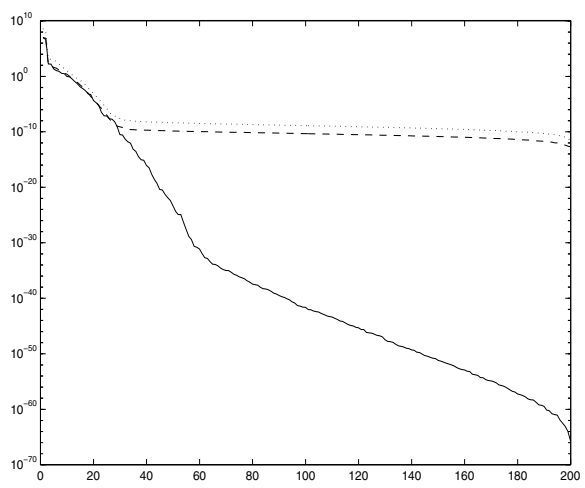

Fig. 24.14. $\cdots \operatorname{svd}\left(\mathcal{G}_{c}\right), \operatorname{o~} \operatorname{svd}\left(\mathcal{G}_{o}\right),-\mathrm{hsv}$

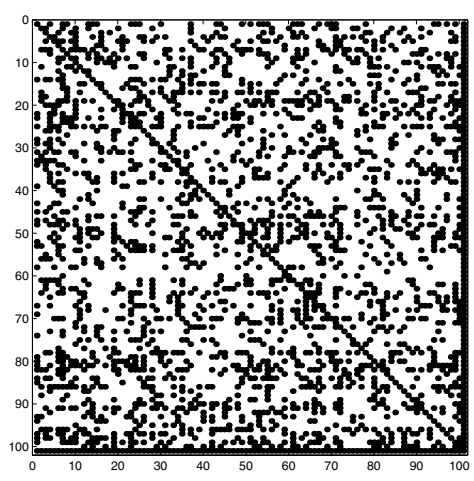

Fig. 24.15. Sparsity of $A$ (RAND) 


\subsection{Building Model}

Mechanical systems are typically modeled as second order differential equations

$$
\left\{\begin{aligned}
M \ddot{q}(t)+D \dot{q}(t)+S q(t) & =B_{q} u(t), \\
y(t) & =C_{q} q(t)
\end{aligned}\right.
$$

where $u(t)$ is the input or forcing function, $q(t)$ is the position vector, and where the output vector $y(t)$ is typically a function of the position vector. Here $M$ is the (positive definite) mass matrix, $D$ is the damping matrix and $S$ is the stiffness matrix of the mechanical system. Since $M$ is invertible, one can use the extended state

$$
x(t)^{T}=\left[q(t)^{T} \dot{q}(t)^{T}\right]
$$

to derive a linearized state space realization

$$
A:=\left[\begin{array}{cc}
0 & I \\
-M^{-1} S-M^{-1} D
\end{array}\right], \quad B:=\left[\begin{array}{c}
0 \\
M^{-1} B_{q}
\end{array}\right], \quad C:=\left[\begin{array}{ll}
C_{q} & 0
\end{array}\right]
$$

or a weighted extended state

$$
x(t)^{T}=\left[q(t)^{T} M^{-\frac{1}{2}} \dot{q}(t)^{T} M^{-\frac{1}{2}}\right]
$$

yielding a more "symmetric" model

$$
A:=\left[\begin{array}{cc}
0 & I \\
-\hat{S} & -\hat{D}
\end{array}\right], \quad B:=\left[\begin{array}{c}
0 \\
\hat{B}_{q}
\end{array}\right], \quad C:=\left[\hat{C}_{q} 0\right]
$$

and where $\hat{D}=M^{-\frac{1}{2}} D M^{-\frac{1}{2}}, \hat{S}=M^{-\frac{1}{2}} S M^{-\frac{1}{2}}, \hat{B}=M^{-\frac{1}{2}} B$ and $\hat{C}=C M^{-\frac{1}{2}}$. When $M$ is the identity matrix, one can recover the original matrices from the linearized model. If this is not the case, those matrices are also provided in the benchmark data.

\subsubsection{Simple Building Model (BUILD-I)}

This is a small model of state dimension $N=48$. It is borrowed from [ASG01].

\subsubsection{Earth Quake Model (BUILD-II)}

This is a model of a building for which the effect of earthquakes is to be analyzed (it is provided by Professor Mete Sozen of Purdue University). The mass matrix $M$ is diagonal and of dimension $N=26394$. The stiffness matrix $S$ is symmetric and has the sparsity pattern given in Figure 24.19.

The damping matrix is chosen to be $D=\alpha M+\beta S$, with $\alpha=0.675$ and $\beta=0.00315$. The matrix $B_{q}$ is a column vector of all ones and $C_{q}=B_{q}^{T}$. No exact information is available on the frequency response and on the Gramians of this large scale system. 


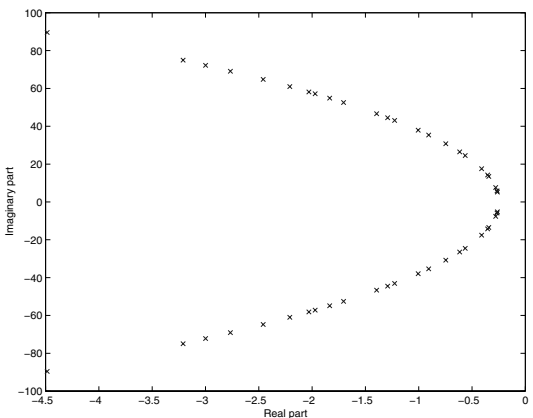

Fig. 24.16. Eigenvalues of $A$ (BUILD-I)

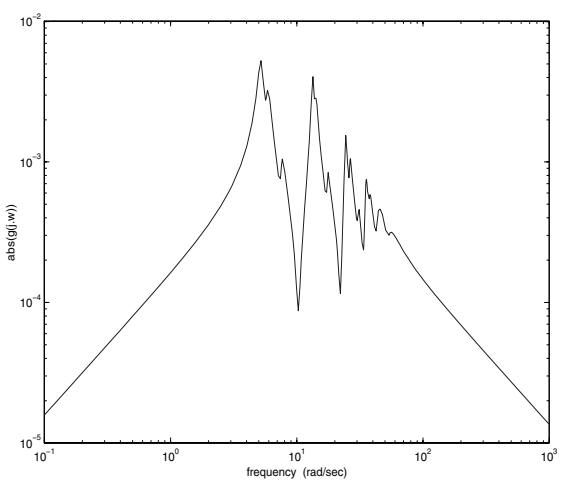

Fig. 24.18. Freq. response (BUILD-I)

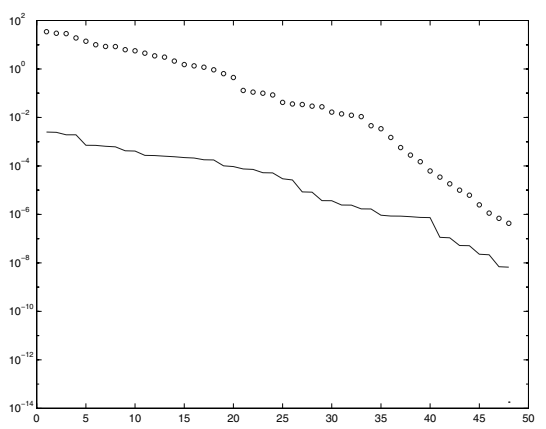

Fig. 24.17. $\cdots \operatorname{svd}\left(\mathcal{G}_{c}\right)$, o $\operatorname{svd}\left(\mathcal{G}_{o}\right),-$ hsv

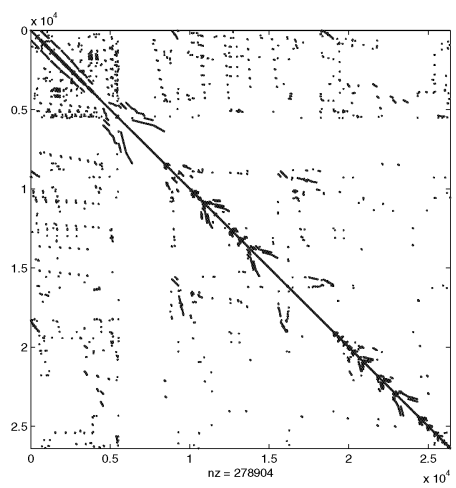

Fig. 24.19. Sparsity of $S$ (BUILD-II) 


\subsubsection{Clamped Beam Model (BEAM)}

The clamped beam model has 348 states, it is obtained by spatial discretization of an appropriate partial differential equation. The input represents the force applied to the structure at the free end, and the output is the resulting displacement. The data were obtained from [ASG01].

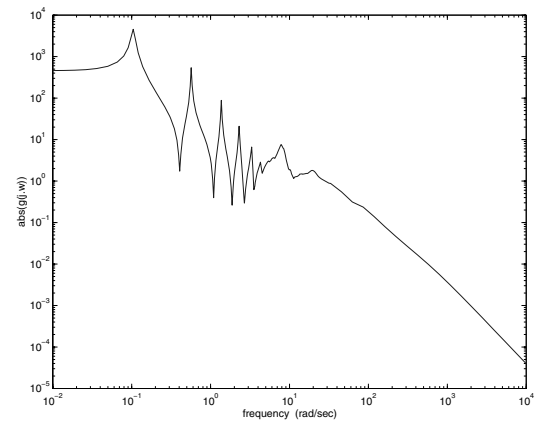

Fig. 24.20. Frequency response (BEAM)

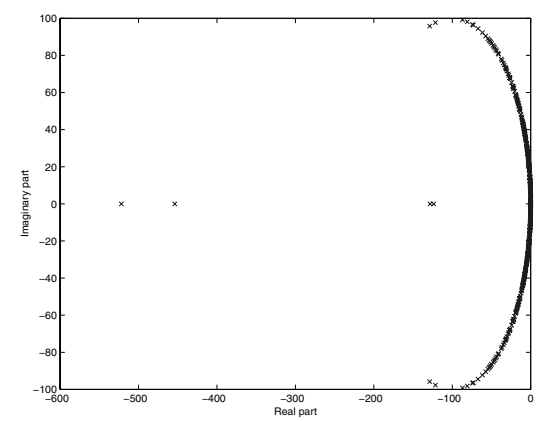

Fig. 24.21. Eigenvalues of $A$ (BEAM)

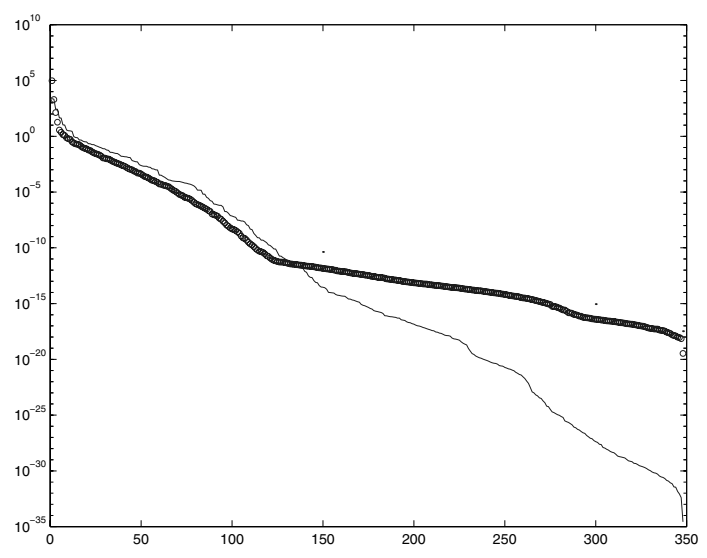

Fig. 24.22. $\cdots \operatorname{svd}\left(\mathcal{G}_{c}\right), o \operatorname{svd}\left(\mathcal{G}_{o}\right),-$ hsv 


\subsection{International Space Station}

This is a structural model of the International Space Station being assembled in various stages. The aim is to model vibrations caused by a docking of an incoming spaceship. The required control action is to dampen the effect of these vibrations as much as possible. The system is lightly damped and control actions will be constrained. Two models are given, which relate to different stages of completion of the Space Station [SAB01]. The sparsity pattern of $A$ shows that it is in fact derived from a mechanical system model.

\subsubsection{Russian Service Module (ISS-I)}

This consists of a first assembly stage (the so-called Russian service module 1R [SAB01]) of the International Space Station. The state dimension is $N=270$.

\subsubsection{Extended Service Module (ISS-II)}

This consists of a second assembly stage (the so-called 12A model [SAB01]) of the International Space Station. The state dimension is $N=1412$.

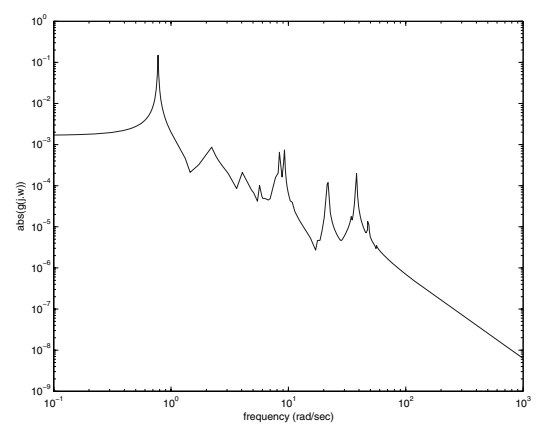

Fig. 24.23. Frequency response (ISS-I)

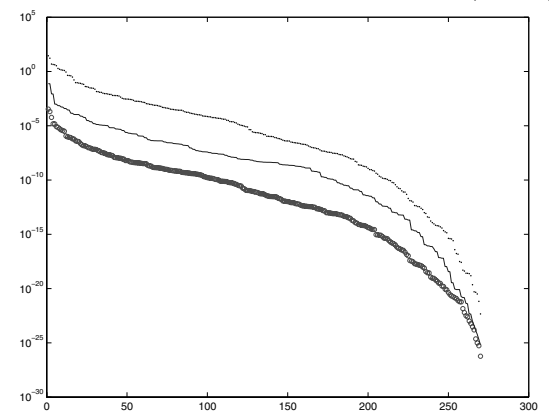

Fig. 24.25. $\cdots \operatorname{svd}\left(\mathcal{G}_{c}\right), o \operatorname{svd}\left(\mathcal{G}_{o}\right),-$ hsv

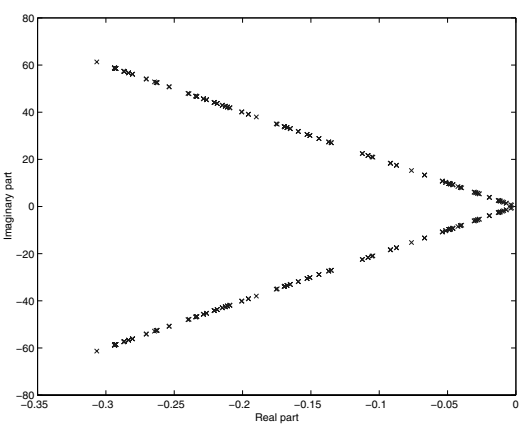

Fig. 24.24. Eigenvalues of $A$ (ISS-I)

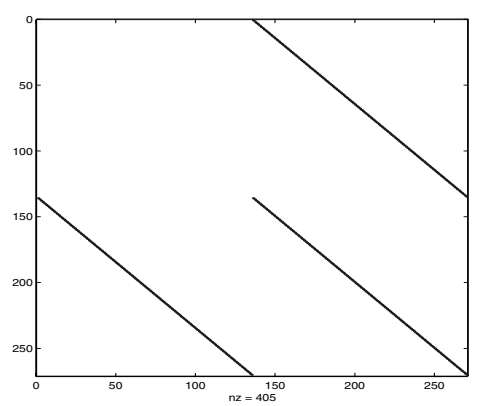

Fig. 24.26. Sparsity of $A$ (ISS-I) 
1st input / 1st output

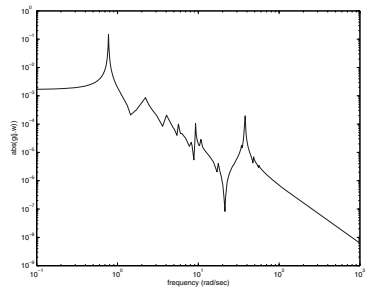

2nd input / 1st output

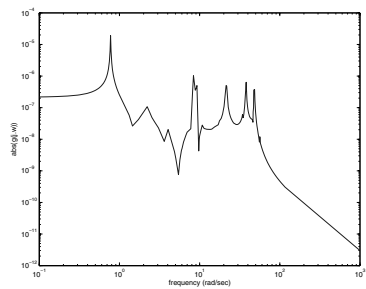

3rd input / 1st output

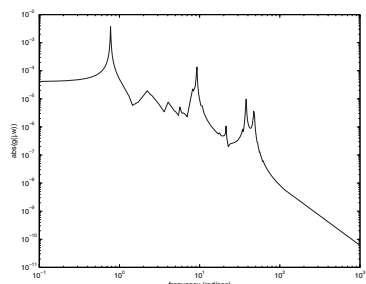

1st input / 2nd output

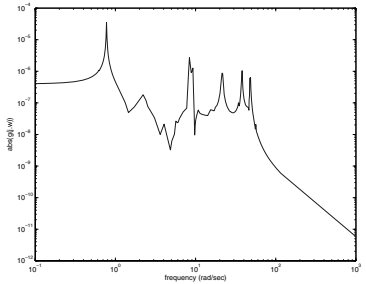

2nd input / 2nd output

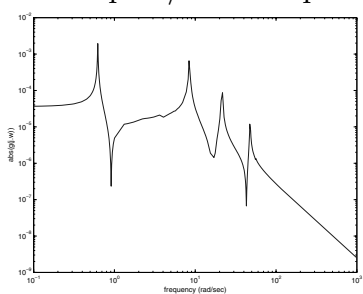

3rd input / 2nd output

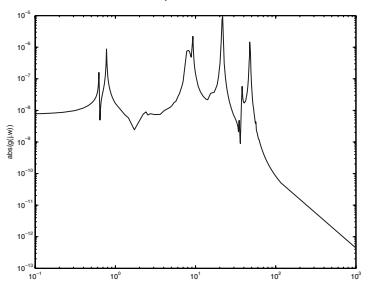

1st input / 3rd output

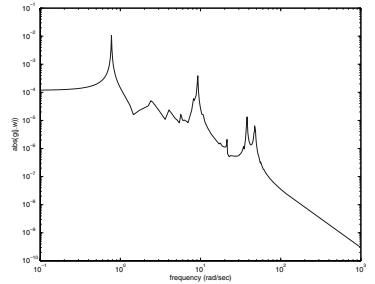

2nd input / 3rd output

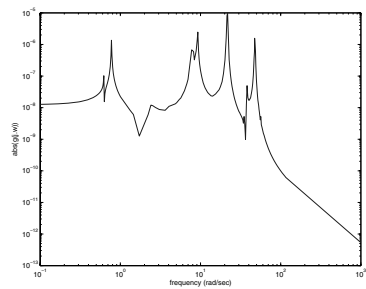

3rd input / 3rd output

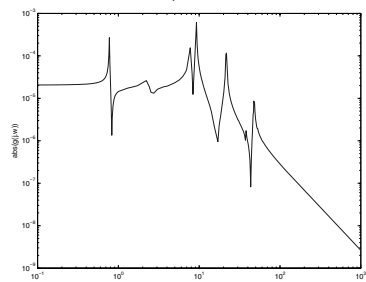

Fig. 24.27. Frequency response of the 3-input 3-output system (ISS-II)

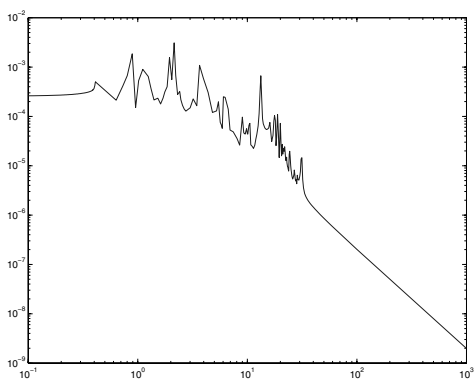

Fig. 24.28. Frequency response (ISS-II)

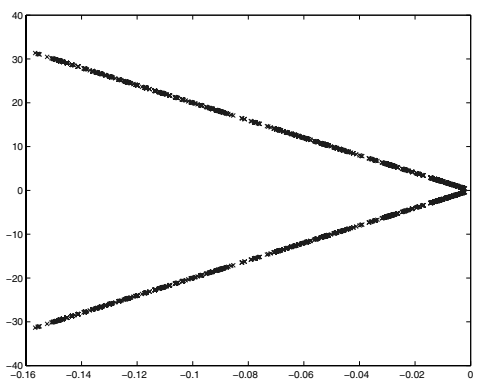

Fig. 24.29. Eigenvalues of $A$ (ISS-II) 


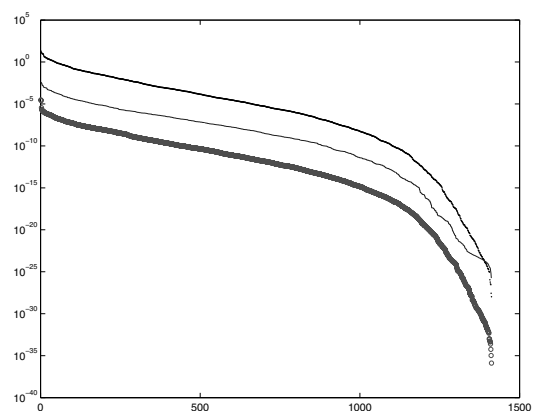

Fig. 24.30. $\cdots \operatorname{svd}\left(\mathcal{G}_{c}\right)$, o $\operatorname{svd}\left(\mathcal{G}_{o}\right),-\mathrm{hsv}$ (ISS-II)

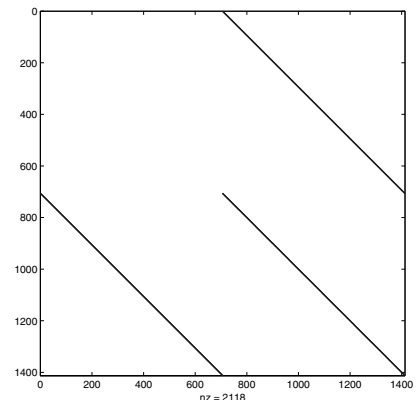

Fig. 24.31. Sparsity of $A$ (ISS-II)
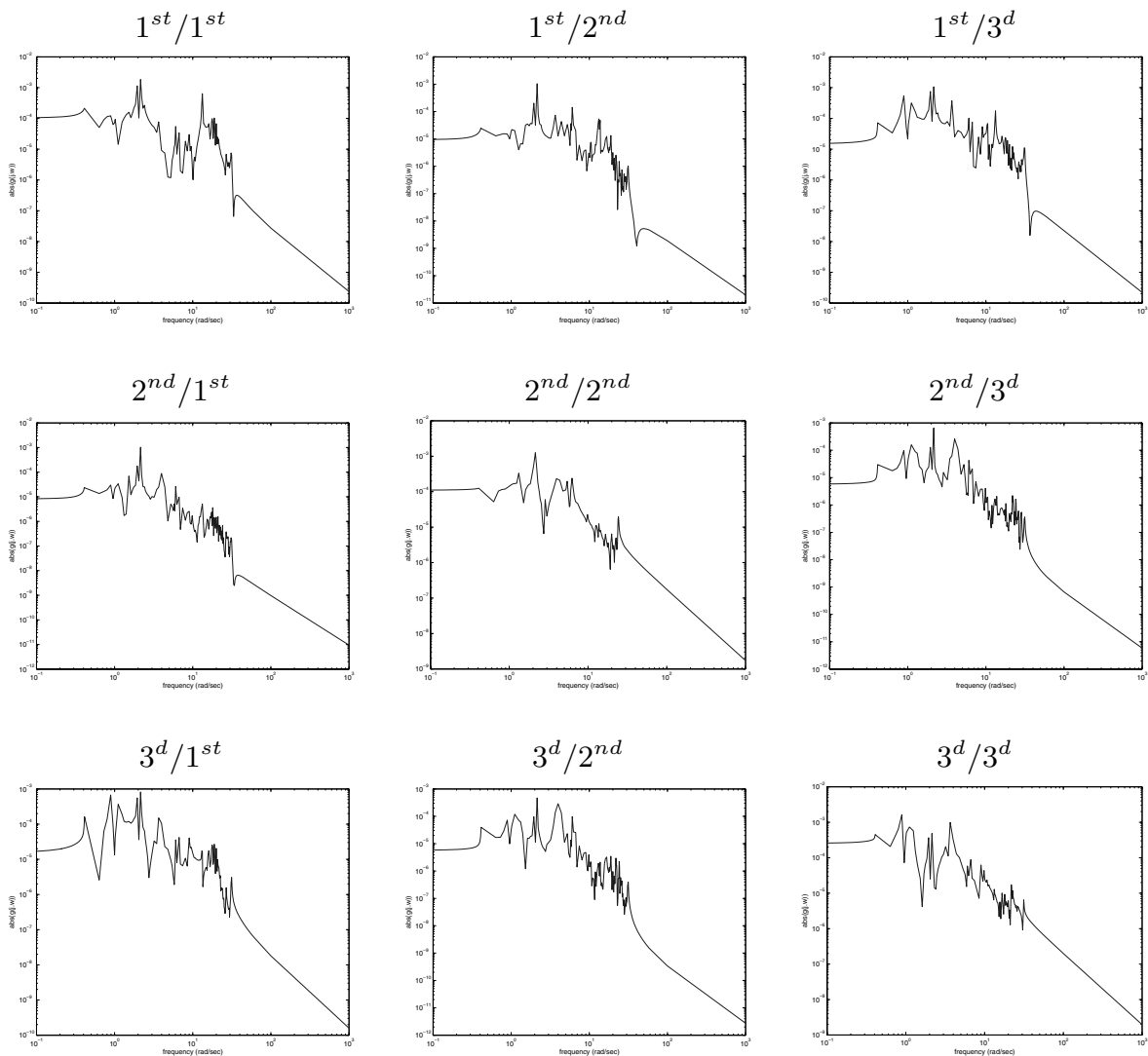

Fig. 24.32. Frequency response of the ISS12A model $\left(i^{\text {th }}\right.$ input $/ j^{\text {th }}$ output). 


\section{Acknowledgment}

We would like to thank all contributors who sent us their examples for inclusion in this report : A. Antoulas, V. De Clippel, B. Farrell, P. Ioannou, M. Sozen and P. Wortelboer. This paper presents research supported by NSF contracts CCR-99-12415 and ITR ACI-03-24944 and by the Belgian Programme on Inter-university Poles of Attraction, initiated by the Belgian State, Prime Minister's Office for Science, Technology and Culture. The scientific responsibility rests with its authors.

\section{References}

[ASG01] Antoulas, A., Sorenson, D. and Gugercin, S.: A Survey of Model Reduction Methods for Large-Scale Systems. Contemporary Mathematics, 280, 193-219 (2001)

[CV02] Chahlaoui, Y. and Van Dooren, P.: A collection of benchmark examples for model reduction of linear time invariant dynamical systems. SLICOT Working Note, ftp://wgs.esat.kuleuven.ac.be/pub/ WGS/REPORTS/SLWN2002-2.ps.Z.

[DeC97] De Clippel, V.: Modèles réduits de grands systèmes dynamiques. Engineering Thesis, Université catholique de Louvain, Louvain-la-Neuve (1997)

[DSB92] Draijer, W., Steinbuch, M. and Bosgra, O.: Adaptive Control of the Radial Servo System of a Compact Disc Player. Automatica, 28(3), 455-462 (1992)

[FI95] Farrell, B.F. and Ioannou, P.J.: Stochastic dynamics of the mid-latitude atmospheric jet. Journal of the Atmospheric Sciences, 52(10), 1642-1656 (1995)

[FI01] Farrell, B.F. and Ioannou, P.J.: Accurate Low Dimensional Approximation of the Linear Dynamics of Fluid Flow. Journal of the Atmospheric Sciences, 58(18), 2771-2789 (2001)

[SAB01] Gugercin, S., Antoulas, A. and Bedrossian, N.: Approximation of the International Space Station 1R and 12A flex models. In: Proc. of the IEEE Conference on Decision and Control, Orlando, Paper WeA08 (2001)

[WSB96] Wortelboer, P., Steinbuch, M. and Bosgra, O.: Closed-Loop Balanced Reduction with Application to a Compact Disc Mechanism. Selected Topics in Identification, Modeling and Control, 9, 47-58, (1996) 